\title{
Weaknesses of a public-key cryptosystem based on factorizations of finite groups
}

\author{
Simon Blackburn * Sean Murphy \\ Information Security Group, \\ Royal Holloway and Bedford New College, \\ University of London, \\ Egham, Surrey TW20 0EX, U.K.
}

\author{
Jacques Stern \\ Laboratoire d'Informatique, \\ Ecole Normale Supérieure, \\ 45, rue d'Ulm, \\ 75230 Paris
}

\begin{abstract}
Recently, Qu and Vanstone have announced the construction of several new public-key cryptosystems based on group factorization. One of these was deacribed at the last AUSCRYPT meeting [2]. We point out a serious weakness of this last system which makes it insecure. Our method only wes elementary algebra.
\end{abstract}

\section{The proposed cryptosystem}

Let $G$ be a finite group. A factorization of $G$ is a sequence $A_{1}, \cdots, A_{\text {, of subsets }}$ of $G$ such that each element $g$ of $G$ can be expressed uniquely as a product

$$
g=g_{s} g_{s-1} \cdots g_{1}
$$

where $g_{i} \in A_{i}$.

The public-key cryptosystem described in [2] uses the additive group $G=Z_{2}^{n}$. Starting from a sequence $\alpha_{1}, \cdots, \alpha_{n}$ of generators, the authors build a sequence $G=G_{0}>G_{1}>\cdots>G_{n / 2}=\{0\}$ of subgroups, where $G_{i}$ is generated by $\left\{\alpha_{2 i+1}, \alpha_{2 i+2}, \alpha_{2 i+3}, \cdots, \alpha_{n}\right\}$. Next, a complete set of coset representatives of $G_{i}$ in $G_{i-1}$

$$
\bar{A}_{i}=\{\bar{\alpha}[i, 0], \bar{\alpha}[i, 1], \bar{\alpha}[i, 2], \bar{\alpha}[i, 3]\}
$$

is chosen, where

$$
\bar{\alpha}[i, j]=j_{1} \alpha_{2 i-1}+j_{2} \alpha_{2 i}+\alpha_{i, j}
$$

$j_{1} j_{2}$ being the binary expansion of $j$ and $\alpha_{i, j}$ a random element of $G_{i}$.

The family $\bar{A}_{i}$ is defined for $i \leq n / 4-1$. For $n / 4 \leq i \leq n / 2-2$, a somehow similar construction is performed, with the difference that four complete sets

$$
\bar{A}_{i, h}=\left\{\bar{\alpha}[i, 0]_{h}, \bar{\alpha}[i, 1]_{h}, \bar{\alpha}[i, 2]_{h}, \bar{\alpha}[i, 3]_{h}\right\}
$$

are built instead of one.

- This author was supported by S.E.R.C. research grant GR/H23719 
Finally, a one-one function $f$ that maps the set $\{1, \cdots, n / 4-1\}$ onto the set $\{n / 4, \cdots, n / 2-2\}$ is chosen and the public key $A_{1}, \cdots, A_{n / 4-1}$ is defined where $A_{i}$ is obtained by (randomly) ordering the set

$$
\bigcup_{\substack{0 \leq j \leq 3 \\ 0 \leq h \leq 3}}\left\{\bar{\alpha}[i, h]+\bar{\alpha}[f(i), j]_{h}\right\}
$$

as

$$
\alpha[i, p] \quad, \quad 0 \leq p \leq 15
$$

A message $m$ of $n-4$ bits is encoded as follows: packing the bits 4 by 4 , one obtains a sequence $m_{1}, \cdots, m_{n / 4-1}$ of hexadecimal digits from which the ciphertext $c$ is computed as:

$$
c=\sum \alpha\left[i, m_{i}\right]
$$

The secret data coming from the construction itself allow decoding: using the decomposition of $c$ in the basis $\alpha_{1}, \cdots, \alpha_{n}$ successively gives, for each $i=$ $1 \cdots n / 4-1$, the corresponding index $h$ of the vector

$$
\bar{\alpha}[i, h]+\bar{\alpha}[f(i), j]_{h}
$$

chosen from $A_{i}$ vis $m_{i}$. But, once all the values $h$ are known, the decomposition of $c$ in the basis $\alpha_{1}, \cdots, \alpha_{n}$ also gives by an easy recursion on $k=n / 4 \cdots n / 2-2$ the missing part $\bar{\alpha}[k, j]_{h}$ of the vector

$$
\bar{\alpha}\left[f^{-1}(k), h\right]+\bar{\alpha}[k, j]_{h}
$$

chosen at level $f^{-1}(k)$. All this can be made quite efficient (see [2]). The value $n=128$ is suggested for practical implementations.

\section{Basic observations}

In [2], it is stated that, although the cryptosystem is a kind of (modular) knapsack, methods using lattice reduction, such as the Lagarias-Odlyzko attack ( [3]) do not apply. We agree with this opinion and we think it remains true even with recent improved versions of this attack such as [1]. Thus, we take another way.

Note that the group $G$ used in section 1 is also a vector space: using Gaussian elimination over the field $Z / 2 Z$, it is easy to compute the dimension of a subspace of $G$ generated by a given family $X$ of vectors and to output a basis of this subspace.

Also, whenever a large family $X$ is chosen in a subspace $F$ of $G$, this family is quite likely to generate the entire subspace. For example, we have:

Theorem 1 If $P$ vectors are chosen independantly at random in a vector space of dimension $K$ over the two element field, the probability that they do not generate the entire space is at most $2^{P-K}$. 
The proof of this result is quite simple: given a subspace of dimension $k-1$, the probability that all choices remain in this subspace is at most $\frac{1}{2}^{P}$. But the number of such subepaces is exactly the number of non-zero linear functionals, i.e. $2^{K}-1$.

From the public key of the above cryptosystem we define

$$
A^{i}=\bigcup_{j>i} A_{i}
$$

and we obeerve the following

Fact: With high probability, $A^{i}$ generates the subspace $G_{i}$, provided $i$ is not too large.

Note that $A^{i}$ contains $16(n / 4-i)$ vectors from the space $G_{i}$, which is of dimension $n-2 i$. Even if the vectors from $A^{i}$ are not really chosen independantly, the above theorem still gives a convincing eatimate of the probability that $A^{i}$ does not generate $G_{i}$, namely $2^{3 n-14 i}$. For $n=128$, this estimate remains below $2^{40}$ up to $i=27$. This leaves out only four values.

Thus, it is fairly clear that some secret information leeks out. In the next section, we will see how to take advantage of this fact.

\section{Cryptanalysis of the system}

From section 2, we know that we can recover from the public data the sequence of subgroups $G_{i}$, for $i$ not too large, say $i \leq i_{0}$. Our cryptanalysis include several steps.

Grouping the elements of $A_{i}$ together, for $i$ not too large. Although the elements of each $A_{i}$ have been scrambled, it is possible to group together the elements

$$
\bar{\alpha}[i, h]+\bar{\alpha}[f(i), j]_{h}
$$

with the same $h$ by using the equivalence relation

$$
u \oplus v \in G_{i}
$$

Since, the $G_{i}$ 's are known up to $i=i_{0}$, the grouping is properly recovered up to $i=i_{0}$ as well. We note that actually, whenever $u$ and $v$ are equivalent elements of $A_{i}$, the sum $u \oplus v$ belongs to $G_{n / 4-1}$. This way, we can collect a fairly large family $Y$ of elements of $G_{n / 4-1}$.

Extending the method to the lest few indices. For $i$ between $i_{0}$ and $n / 4-1$, we have not been able so far to compute accurately $G_{i}$ because the sample $A^{i}$ of elements of $G_{i}$ was not large enough. Now, if we add to $A^{i}$ the set $Y$ that has been computed at the end of the last paragraph, then we see that we obtain a generating family for $G_{i}$. From this, we can aleo perform the correct grouping of $A_{i}$. 
Recovering the secret permutation $f$. We work again with the equivalence relation on $A_{i}$ defined above and, this time, we use the fact that, whenever $u$ and $v$ are equivalent elements of $A_{i}$, the sum $u \oplus v$ belongs to $G_{f(i)-1}$ (and not only to $G_{n / 4-1}$ as was observed above). We let $B_{i}$ be the set of sums $u \oplus v$ obtained from equivalent elements of $A_{i}$. Each $B_{i}$ contains 24 elements. We define:

$$
B^{i}=\bigcup_{j \neq i} B_{i}
$$

fact:

i) if $f(i)=n / 4$ then, with high probability, $B^{i}$ generates the subspace $G_{n / 4}$

ii) otherwise, with high probability, $B^{i}$ generates the subspace $G_{n / 4-1}$

This is because, we have, in each case a very large family of members of the corresponding space.

From the fact, it follows that we can recover both $f^{-1}(n / 4)$ and $G_{n / 4}$ by computing the dimension of all the spaces generated by the various families $B^{i}$. A recuraive procedure will then achieve the same for $f^{-1}(n / 4+j)$ and $G_{n / 4+j}$ : this procedure uses the same argument, the family $B_{i}$ being restricted to those indices $i$ for which $f(i)$ is not yet known (i.e. is $\geq n / 4+j$ ).

Note that, at atep $j$, we have $24(n / 4-j-2)$ elements of a subspace of dimension $n / 2-2 j$ or $n / 2-2 j+2$. Using the estimate of theorem 1 , we that the probability of error remains quite small even for the last significant case $(j=n / 4-3)$, for which it is below $2^{-16}$. Still, there is a slight chance that $f^{-1}(n / 4+j)$ is not correctly computed for say the last two or three values. This iseue will be addressed specifically when we turn to decoding.

Decoding. Given the ciphertext $c$, we first apply the following procedure:

for $i:=1$ to $n / 4-1$ do

begin

pick any $u$ in $A_{i}$ such that $c \oplus u$ belongs to $G_{i}$

$u[i]:=u$; return $u[i]$;

$c:=c \oplus u$

end;

return $c$

At the end of the procedure, we have reduced the posible choices of the unique element of $A_{i}$ that contributes to the sum

$$
c=\sum \alpha\left[i, m_{i}\right]
$$

to a subset of each $A_{i}$ consisting of the four elements equivalent to the vector $u$ [i] returned at step $i$. We denote this subset by $\tilde{A}_{i}$. Next, we apply the following. for $i:=n / 4$ to $n / 2-2$ do

begin

pick any $v$ in $\tilde{A}_{f-1(i)}$ such that $c \oplus u[i] \oplus v$ belongs to $G_{i}$

$v[i]:=v ;$ return $v[i]$;

$c:=c \oplus u$;

end 
The value of $v[i]$ returned at step $i$ is the vector $\alpha\left[i, m_{i}\right]$ of the sum

$$
c=\sum \alpha\left[i, m_{i}\right]
$$

This gives the plaintext $m$. Efficient implementations using decoding matrices can be implemented as in [2].

As observed above, there is a small chance that a mistake occurs for two or three values $i=f^{-1}(n / 4-2), i=f^{-1}(n / 4-3)$, etc. This can be corrected by exhaustive search. Note that, since the mistake comes from the attack (and not from the ciphertext), the proper value of $f$ can be recovered from a few decoding computations.

\section{Conclusion}

We have pointed out a serious weakness in the system proposed in [2]. Furthermore, we do not feel simple modifications of the system can restore its security. For example, it is quite possible to changer the order of the $A_{i}$ 's but the correct order can be recovered by computing dimensions with the same method we used to disclose $f$.

\section{References}

1. M. J. Coster, A. Joux, B. A. LaMacchia, A. M. Odlyzko, C. P. Schnorr and J. Stern, Improved low-density oubeet sum algorithms, Computational Complexity, to appear.

2. M.Qu, S. A. Vanstone, New public-key cryptosystems based on factorizations of finite groups, AUSCRYPT'92, preproceedings page 12.7-12.12.

3. J. C. Lagarias and A. M. Odlyzko, Solving low-density subet sum problems, J. Assoc. Comp. Mach. 32 (1985), 229-246. 